\title{
Morphology and rheology of cellulose nanofibrils derived from mixtures of pulp fibres and papermaking fines
}

\author{
Jérôme Colson • Wolfgang Bauer • \\ Melanie Mayr • Wolfgang Fischer • \\ Wolfgang Gindl-Altmutter
}

Received: 1 March 2016/Accepted: 13 June 2016/Published online: 16 June 2016

(C) The Author(s) 2016. This article is published with open access at Springerlink.com

\begin{abstract}
The rheological behaviour of homogenised fibres originally having different lengths was evaluated. For this purpose, mixtures of pulp fibres and fines were fibrillated mechanically without pretreatment and characterised with regard to morphology and viscosity. It was found that, for all samples, a similar number of homogenisation passes was needed to reach a viscosity plateau. However, the value of the final viscosity differed significantly: homogenised suspensions derived from fines achieved only about $60 \%$ of the viscosity of suspensions derived from pulp. Already after a few homogenisation cycles, no differences between the samples could be measured using optical devices, indicating that fibrillation on the nanometre scale was responsible for the distinct rheological behaviours. Atomic force microscopy
\end{abstract}

Electronic supplementary material The online version of this article (doi:10.1007/s10570-016-0987-x) contains supplementary material, which is available to authorized users.

J. Colson $(\bowtie) \cdot$ W. Gindl-Altmutter

Department of Materials Sciences and Process Engineering, Institute of Wood Technology and Renewable Materials, University of Natural Resources and Life Sciences Vienna, Konrad-Lorenz-Straße 24, 3430 Tulln, Austria

e-mail: jerome.colson@boku.ac.at

W. Bauer · M. Mayr · W. Fischer Institute of Paper, Pulp and Fibre Technology, NAWI Graz, Graz University of Technology, Inffeldgasse 23, 8010 Graz, Austria measurements indicated significantly reduced fibril lengths for the suspensions derived from fines, which explains their reduced viscosity.

Keywords Homogenisation · Rheology · Pulp · Fibre length $\cdot$ Fines $\cdot$ Cellulose nanofibrils

\section{Introduction}

Nanocellulose is a material consisting of very small diameter cellulose fibrils. It is, for example, secreted extracellularly by several bacteria [the most efficient being acetobacter xylinum (Jonoobi et al. 2015)] and is then called bacterial cellulose. Different technological processes also lead to the production of nanocellulose. Usually, the raw material is wood pulp which undergoes an intensive mechanical treatment by highpressure homogenisation, microfluidisation, or grinding. Alternative starting materials such as various kinds of residue from the food industry (Leitner et al. 2007) or from biotechnological conversion processes (Henniges et al. 2014) also provide useful fibrillated material requiring significantly less input of mechanical disintegration energy compared to that needed for fibrillating wood pulp fibres. In the pulping and papermaking process, not only long fibres suitable for paper are produced, but depending on the technology used, a certain amount of fine material ("fines") is also obtained. Fines may be classified into primary fines, 
which originate from the pulping process and consist of mostly parenchyma and ray cells or vessel elements, and secondary fines, which are produced during mechanical refining of pulp. Secondary fines consist of various cell wall fragments. The definition of fines may slightly vary depending on the standard used: they are e.g. defined as the fibres passing a round hole $76 \mu \mathrm{m}$ in diameter (TAPPI 1994; ISO 2012) or as the particles shorter than $200 \mu \mathrm{m}$ (ISO 2014). For practical reasons, the latter definition was used in this study.

The properties of cellulose nanofibrils obtained after mechanical fibrillation may vary depending on several factors, e.g. the used raw material (Zimmermann et al. 2010; Syverud et al. 2011), the chosen pretreatment (Henriksson et al. 2007; Pääkko et al. 2007) and many others. Among the influencing factors, fibre size distribution of the raw material is also important. In the past, rheological characterization of synthetic fibre distributions has already been of interest (Powell 1991; Djalili-Moghaddam and Toll 2006). Rheology of natural fibres has also already been experimentally studied. For example, Silveira, Ferreira and Lobo (Silveira et al. 2002; Ferreira et al. 2003; Silveira et al. 2003) investigated the influence of temperature, $\mathrm{pH}$, solid content, fibre length and fibre mix on the flow behaviour of cellulose fibres from bleached eucalyptus and pine kraft pulps. The material used in these studies was not homogenised.

Other studies (Lasseuguette et al. 2008; AgodaTandjawa et al. 2010; Iotti et al. 2011; Naderi et al. 2014; Li et al. 2015) focused on the rheological behaviour of different kinds of nanocelluloses. It has been shown that the viscosity of cellulose nanofibre suspensions decreases with increasing shear rate, which corresponds to a typical shear-thinning behaviour. However, in some shear rate ranges, the

Table 1 Sample characteristics

\begin{tabular}{lcc}
\hline Sample name & Fines content $(\%)$ & Long fibre content $(\%)$ \\
\hline F0 & 0 & 100 \\
F25 & 25 & 75 \\
F50 & 50 & 50 \\
F75 & 75 & 25 \\
F100 & 100 & 0 \\
\hline
\end{tabular}

opposite behaviour was observed. This phenomenon was explained by the entanglement of fibres in the suspension, causing a shear-induced structure. At higher shear stress (i.e. higher shear rate), this structure would break, causing the viscosity to decrease again. Furthermore, it has been proven by Takana et al. (Tanaka et al. 2014) that, when the solid content is lower than a certain critical value (dilute regime), the shear-viscosity of cellulose nanofibrils suspensions is directly related to their length, assuming a uniform width. In this publication, it was pointed out that the equation used for the evaluation of the viscosity-average lengths (Doi and Edwards 1986) was established for rigid, rod-like particles, and has still to be adapted for semi-flexible cellulose nanofibrils. For cellulose nanocrystals, which can be considered as rigid rods, the calculated aspect ratio values were always in accordance with the experimental determinations by atomic force microscopy (AFM) ( $\mathrm{Li}$ et al. 2015) or transmission electron microscopy (TEM) (Iwamoto et al. 2013).

The interest in the rheological behaviour of nanocellulose suspensions is not only a purely theoretical one: in some applications (e.g. coatings), the viscosity of the final product is of crucial importance (Lindström et al. 2015). Even though it is not yet possible to completely control the rheology of a system containing nanocellulose (Naderi and Lindström 2015), it is a promising, sustainable additive. That is why efforts have to be made to better understand the influence of nanocellulose in suspensions subjected to flow. In the present study, we evaluate cellulose nanofibrils derived from secondary pulp fines and compare the differences in morphology and rheology of cellulose nanofibrils derived from different mixtures of fibres and fines.

\section{Materials and methods}

\section{Materials}

Samples used in the present study were never-dried bleached softwood sulphite pulp (F0) and never dried bleached sulphite fines (F100) kindly provided by a project consortium. Solid contents as received were 12 and $20 \%$, respectively. Both samples were ashfree. 
Sample washing and fines separation

To obtain pulp samples consisting purely of long fibres without fines, the original pulp sample was treated as follows: Its solid content was first brought to $1 \%$ by dilution with tap water and the pulp was stirred for $10 \mathrm{~min}$. It was then fractionated using a lab pressure screen implemented at Graz University of Technology, at a rotor speed of $17 \mathrm{~m} \mathrm{~s}^{-1}$, a flow rate of $121 \mathrm{~min}^{-1}$ and an overpressure of about 1 bar. The screen had a hole diameter of $100 \mu \mathrm{m}$. The system was operated at $22{ }^{\circ} \mathrm{C}$. The inlet flow was divided into two outlet flows: the reject-flow, containing the washed pulp (free of fines) having a solid content of approximately $2 \%$, and the accept-flow, containing only fines with a solid content of around $0.02 \%$.

Whereas the solid content of the reject flow suits the needs of the present study, the solid content of the accept-flow is very low. The production of a sufficient amount of pure fines to perform the planned sample preparation (fibrillation) and measurements using this pressure-screen method is extremely time consuming. For these reasons, it was decided to use a thicker fines suspension available at another sampling point in the paper machine circulation system to perform the experiments described in this paper.

\section{Fibrillation}

Samples as received were brought to a solid content of $0.5 \%$ and stored at $5{ }^{\circ} \mathrm{C}$ for a few days to ensure complete swelling of the fibres. These samples were then used to produce mixtures with different fines/long fibre contents (compare Table 1). In the next step the different mixtures were ground using a Masuko MKCA6-2 J CE supermasscolloider (Masuko ${ }^{\circledR}$, Japan) at $1500 \mathrm{rpm}$ and a gap clearance of $-50 \mu \mathrm{m}$. This step was repeated 5 times. Samples were subsequently homogenised with an APV1000 $\left(\mathrm{SPX}^{\circledR}\right.$, USA) homogeniser. To avoid getting the fibres stuck in the homogeniser, the pressure was gradually increased: In the first cycle, fibres were passed through the homogeniser without applying any pressure. In the second cycle a pressure of 100 bar and in the third one a pressure of 400 bar was applied. Homogenizing pressure in the following passes was held constant at 800 bar. The first pass at 800 bar was considered as pass number one in the graphs presented in the "Results and Discussion" section. Samples (around $50 \mathrm{ml}$ ) were taken from the suspension after different numbers of homogenisation passes and stored in a refrigerator until use.

\section{Solid content correction}

A few millilitres of each sample taken during the homogenisation were poured in an aluminium cup, weighed and placed into an oven at $103{ }^{\circ} \mathrm{C}$. After complete evaporation of the water, the dry samples were taken out of the oven and placed in a desiccator until they reached room temperature. Their mass was then recorded again, and the solid content was calculated as follows:

$s=\frac{m_{d r y}}{m_{\text {wet }}} * 100$

where $s$ is the solid content in $\%, m_{w e t}$ and $m_{d r y}$ are the mass of the wet and of the oven-dried sample in [g], respectively.

Previous studies have shown that solid content has a significant influence on viscosity (Silveira et al. 2002; Derakhshandeh et al. 2011). In the present study, a systematic increase in solid content after each homogenisation cycle due to evaporation of water was noticed (compare S1 in supplementary data) and had to be compensated.

The target solid content was set $(0.5 \%$ for the first, $1 \%$ for the second measurement series). Samples with a solid content higher than the target were diluted with the appropriate amount of water. Samples with a lower solid content were centrifuged for $10 \mathrm{~min}$ at 10,000 rpm (Z36HK, Hermle Labortechnik GmbH). The excess amount of water was removed with a syringe and the fibres were resuspended using a laboratory vortex mixer.

Viscosity measurements

All measurements were performed with a cone-andplate rheometer (CS50, Bohlin Instruments) equipped with a thermostat set to $20^{\circ} \mathrm{C}$. The cone diameter was $4 \mathrm{~cm}$, the cone angle $4^{\circ}$, and the truncation gap $150 \mu \mathrm{m} .1 .5 \mathrm{ml}$ of the fibre suspension was poured on the plate with a syringe. Measurements at constant shear rate $\left(20\right.$ and $\left.200 \mathrm{~s}^{-1}\right)$ were performed for each sample. Before starting the measurement, equilibration at the same shear rate was performed for $10 \mathrm{~s}$. A cycle of averaging for $10 \mathrm{~s}$ followed by a 
measurement pause of $3 \mathrm{~s}$, the cone still rotating at the given shear rate, was then performed 10 times. The viscosity value used for further calculations was determined by discarding the first 2 measurement cycles and averaging the individual values obtained in the 8 other cycles. As each individual sample could be measured within a short time (140 s), evaporation was considered negligible and no solvent trap was used.

Fibre characteristics

A L\&W Fiber Tester Plus (Lorentzen \& Wettre) having a resolution of $3.3 \mu \mathrm{m} /$ pixel was used to measure the fibre length distributions of the different samples. Furthermore the fines content [according to the standard implemented in the device, ISO 16065-2 (ISO 2014)] was evaluated. For each measurement, approximately $0.1 \mathrm{~g}$ of dry fibres diluted in $150 \mathrm{ml}$ of deionised water was used. The measurement was stopped after 72,000 particles were detected. Two replicates were measured for each sample, followed by a washing step with distilled water after each measurement.

\section{Atomic force microscopy (AFM)}

Samples were diluted to approximately $0.001 \%$. Two drops were given on a mica plate and dried at $103{ }^{\circ} \mathrm{C}$ for $15 \mathrm{~min}$. This step allowed obtaining single fibres sticking on the mica surface. The samples were imaged with an AFM (Dimension Icon, Bruker) using an OTESPA cantilever. Gwyddion 2.42 was used to process the output files from the AFM.

\section{Results and discussion}

Viscosity

The influence of the number of homogenisation passes on viscosity is shown in Fig. 1. From the figure it is apparent that no results could be obtained for the first homogenisation passes if the initial suspension was containing a low amount of fines. In these cases, clearly visible millimetre-sized fibre flocs were expelled from the side of the cone-and-plate system, sometimes causing the rotation of the cone to slow down or even completely stop. When this happened, the measurement was aborted and it was not taken into account. For fines contents of 100,75 , or $50 \%$, the viscosity increases with the number of homogenisation passes, until a plateau value is reached after approximately 20 cycles. This value is in accordance with the number of passes used by our group to obtain nanocellulose from pulp (Veigel et al. 2012; GindlAltmutter et al. 2015). For fines contents of 25 and $0 \%$ (F25 and F0), an increase in viscosity could not be observed because of fibre flocculation. Once these samples were sufficiently homogenised, their

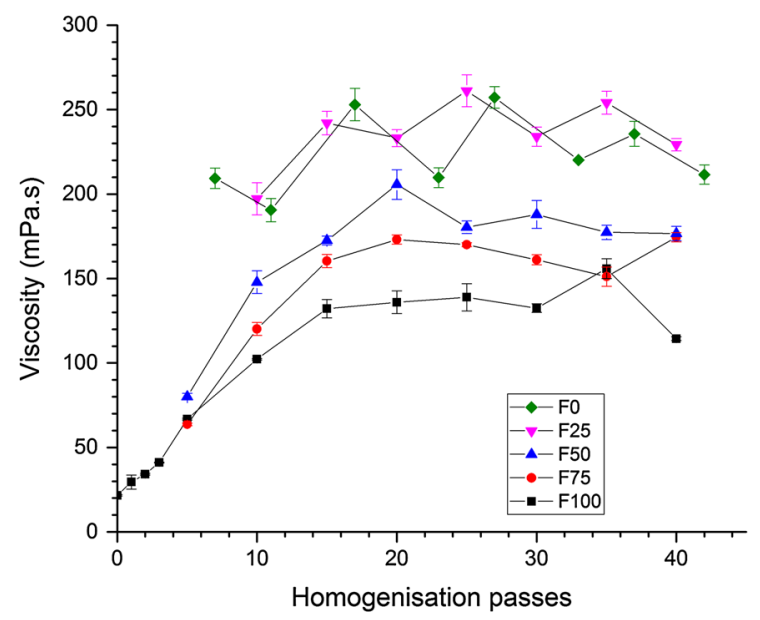

Fig. 1 Viscosity along the homogenisation process for all samples at a shear rate of $20 \mathrm{~s}^{-1}$ and a solid content of $0.5 \%$. Error bars indicate the standard deviation of the rheometer. The lines are a guide to the eye

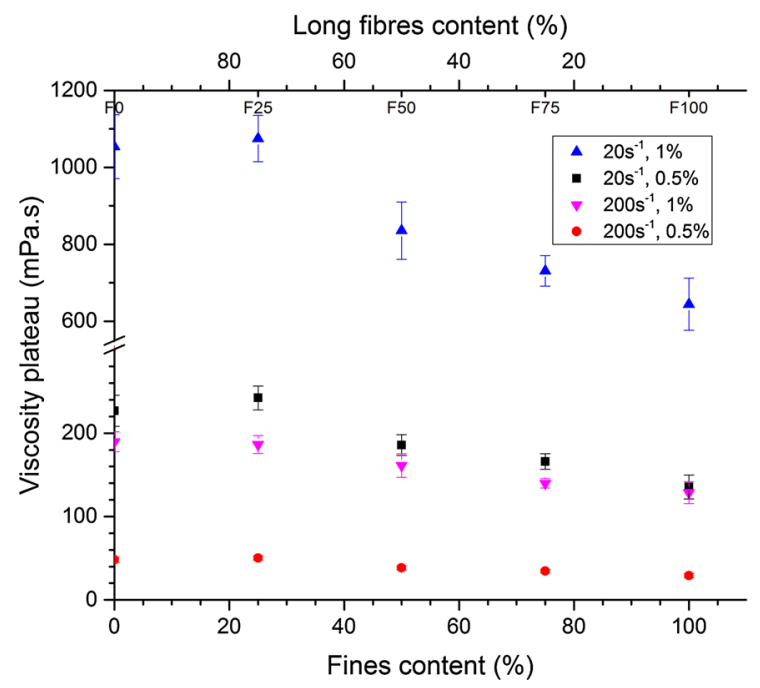

Fig. 2 Value of the viscosity plateau for different shear rates and solid contents 

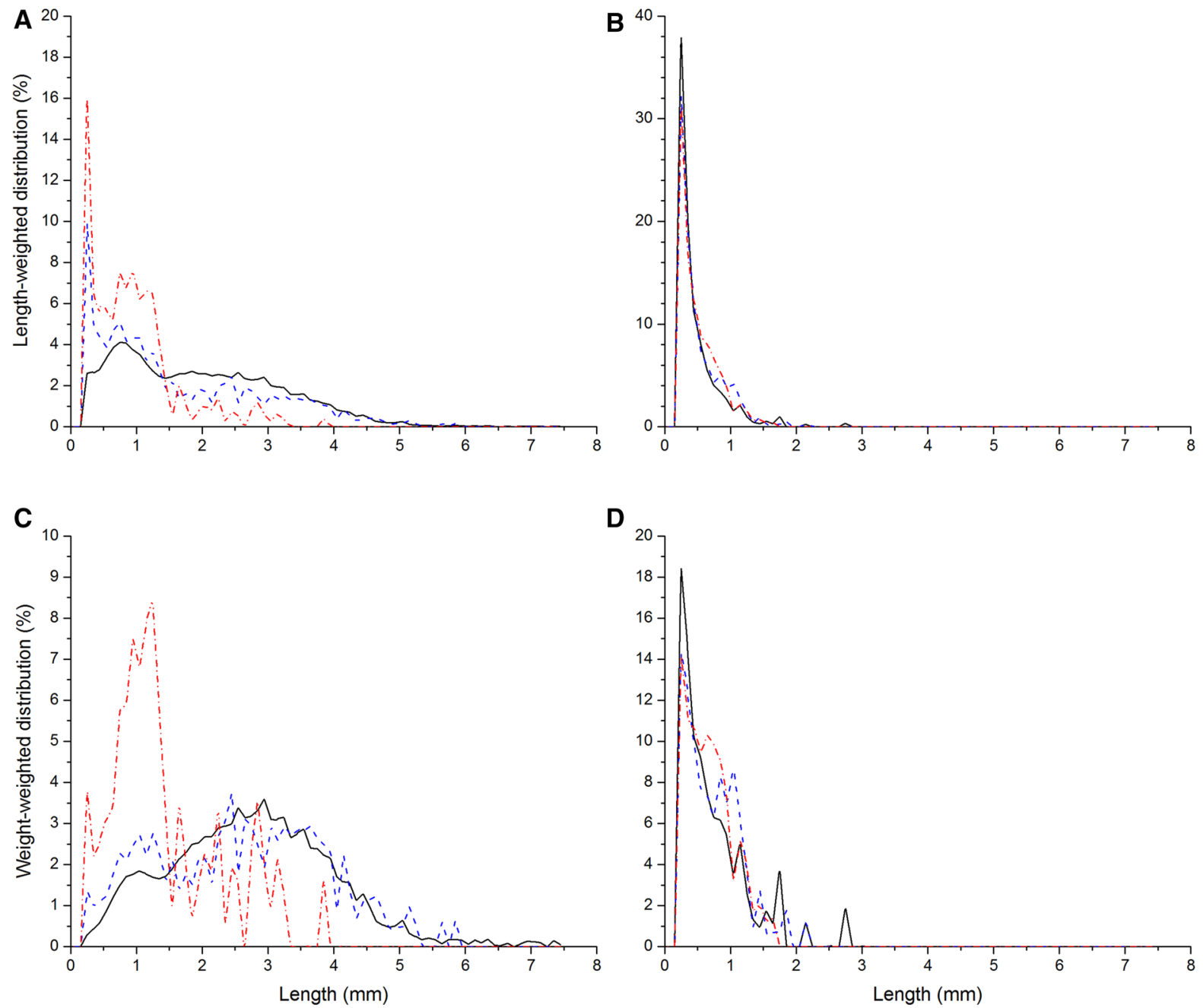

Fig. 3 Length-weighted and weight-weighted length distribution of F0 (plain), F50 (dashed) and F100 (dash-dotted), unprocessed (a, c) and after 10 homogenisation passes $(\mathbf{b}, \mathbf{d})$

viscosity could be measured. The values obtained are oscillating between 200 and $260 \mathrm{mPa}$ s. No reason could be found for this behaviour. As for the other samples, a plateauing was also assumed in this case. However, due to the low amount of samples measured, this cannot be clearly stated here.

The above mentioned behaviour (increase in viscosity and subsequent stagnation after 20 passes) was reproducible for all shear rates and solid contents used in the present study (raw data from the measurements is available in supplementary information S3). Figure 2 shows the influence of the long fibre content, shear rate and solid content on the value of the viscosity plateau. To determine this value, viscosity values measured for the last five data points of Fig. 1 were averaged for each sample type. As it was expected, a higher solid content causes a higher viscosity, whereas a higher shear rate causes a lower viscosity. The latter is due to the shear-thinning behaviour of fibre suspensions, which is well known and has already been studied for several types of fibres (Mallick 2008; Derakhshandeh et al. 2011; Iotti et al. 2011; Rezayati Charani et al. 2013; Naderi et al. 2014). For all combinations of shear rate to solid content, the average plateau value first increases slightly and then steadily decreases when the fines content exceeds $25 \%$. This result is the exact opposite to what has been found with similar measurements on 


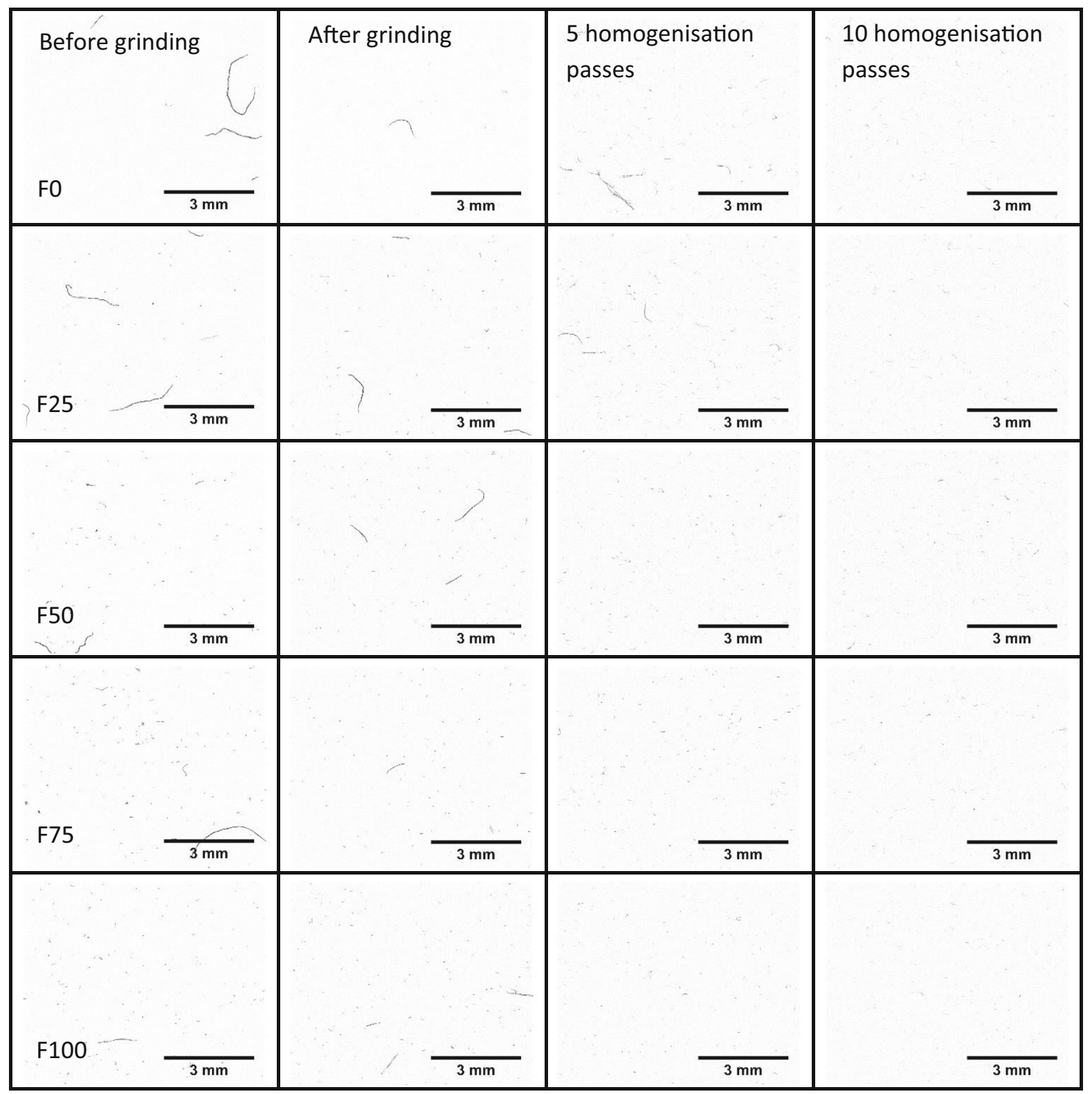

Fig. 4 Images of samples measured with the Fibertester. Text on the left side refers to the fines content, text on the top refers to the number of grinding and homogenisation passes

hard sphere suspensions, where a viscosity minimum was observed at a content of $25 \%$ of small spheres (Parkinson et al. 1970). This indicates that the long fibres, because of higher fibril length, are able to form network structures having a high resistance to flow. The addition of fibres with shorter fibrils will break this network structure and decrease the interactions in the flow, resulting in a lower viscosity.
Numerical models have already been established to calculate the rheology of rigid, rod-like particle suspensions (Powell 1991). However, in the case of natural fibres, flexibility has to be taken into account and these models are no longer valid. Moreover, the surface chemistry of natural fibres is very heterogeneous (contrarily to e.g. carbon or glass rods). For fibres subjected to homogenisation, even more 
Table 2 Length weighted fines content $C f$ and length weighted mean fibre length $\bar{L}$ before and after grinding

\begin{tabular}{lccllc}
\hline Sample & \multicolumn{2}{l}{ Before grinding } & & \multicolumn{2}{c}{ After grinding } \\
\cline { 2 - 3 } & $\mathrm{C}_{\mathrm{f}}(\%)$ & $\bar{L}(\mu \mathrm{m})$ & & $\mathrm{C}_{\mathrm{f}}(\%)$ & $\bar{L}(\mu \mathrm{m})$ \\
\hline F0 & 3.4 & 1900.5 & & 12.4 & 1852.1 \\
F25 & 35.4 & 1152.2 & & 53.6 & 624 \\
F50 & 60.55 & 686.5 & & 66.15 & 469.5 \\
F75 & 75.4 & 358.5 & & 80.45 & 249 \\
F100 & 82.7 & 222.5 & & 90.45 & 125 \\
\hline
\end{tabular}

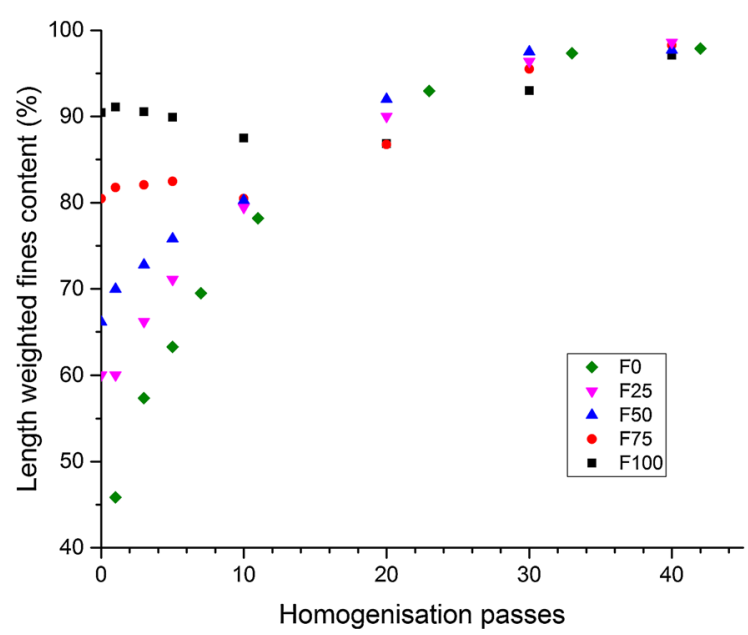

Fig. 5 Length-weighted fines content during the homogenisation process

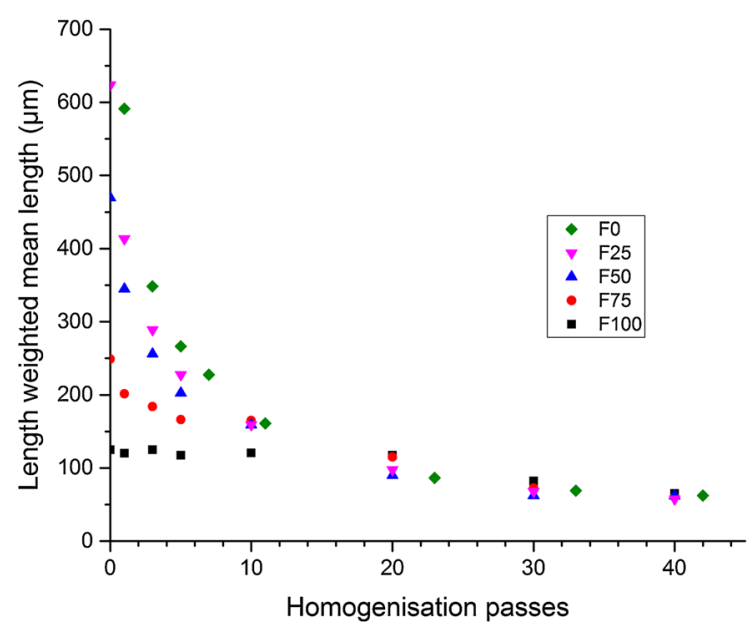

Fig. 6 Length-weighted mean fibre length of the different samples during the homogenisation process complex interactions due to fibrillation of the fibres on the nanometre scale have to be considered. All these parameters make it very difficult to define and quantify the interactions occurring in the suspension subjected to flow. The high complexity of this topic could explain why models of the flow behaviour of highly fibrillated fibres are often valid only under given conditions, e.g. in a defined shear rate range (Puisto et al. 2012).

\section{Fiber tester}

The weight-weighted and length-weighted length distributions of unprocessed and homogenised samples F0, F50 and F100 as measured with the L\&W Fiber Tester Plus can be seen in Fig. 3. As expected, fibre length distribution changes gradually during the homogenisation process. The images taken with the camera of the Fibertester and shown in Fig. 4 support this observation. For the full set of samples including all mixtures of fibres and fines as well as several grinding and homogenisation steps, the reader is referred to supplementary data $\mathrm{S} 2$.

After 10 passes in the homogeniser, a differentiation between the different samples from the images is impossible due to the insufficient resolution of the device. It should be mentioned that the grinding pretreatment influences the mean fibre length as well as the fines content, as shown in Table 2. This table also shows that fines could not be totally removed from the sample F0 and are accounting for $3.4 \%$ of the total fibre length.

The length-weighted fines content is shown in Fig. 5. The fines content of all samples is converging towards $100 \%$. In the first homogenisation passes, the fines content of F100 decreases slightly, which means that the relative total length of fibres longer than $200 \mu \mathrm{m}$ increases. At a first glance, this result may seem contradictory. It can however be quite easily understood if one considers the fibrillation mechanism in the homogenizer, where fibre shortening and fibrillation occur simultaneously (Turbak et al. 1983; Gharehkhani et al. 2015). A long fibre can be shortened several times before it is considered as fine material. In the first homogenisation passes, long fibres will be separated into several shorter fibres which still will not be classified as fines. Depending on the amount and the length of fibres longer than $200 \mu \mathrm{m}$ 

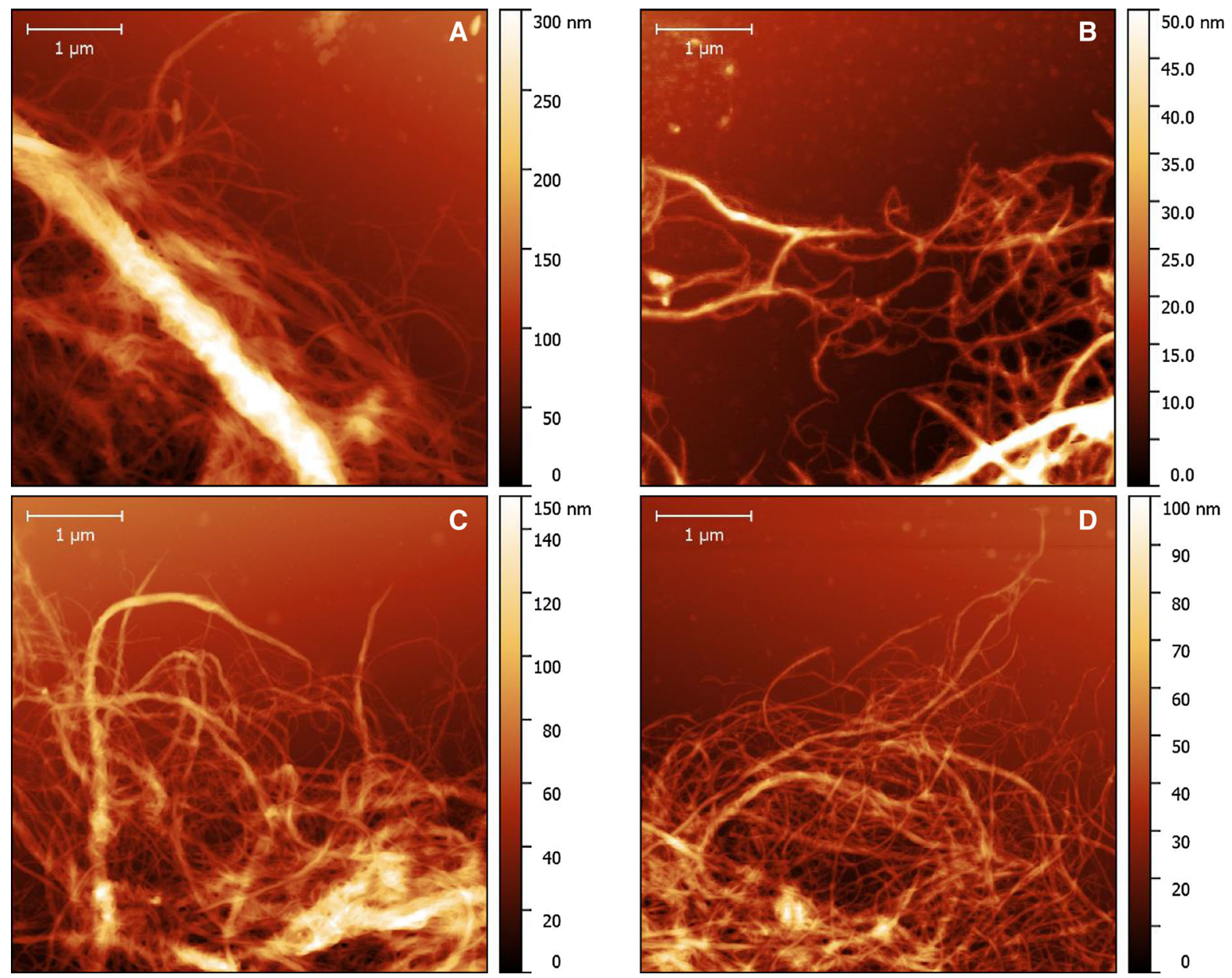

Fig. 7 Topographic AFM scans of F100 after 15 (a) and 35 (b) homogenisation passes and F0 after 15 (c) and 35 (d) homogenisation passes. Scan size is $5 \mu \mathrm{m} \times 5 \mu \mathrm{m}$ in all cases

initially present in the suspension, this may cause the relative total length of long fibres to increase.

The mean fibre length (length-weighted) is shown in Fig. 6. It is converging towards a limit value of around $50 \mu \mathrm{m}$ for all samples. For the same reason as mentioned above, the mean length is constant or even slightly increasing in the first homogenisation passes for sample F100. Moreover, all samples are close to the limit value after 20 homogenisation passes, independently of the mean fibre length before the homogenisation. This confirms the conclusions drawn from the viscosity measurement.

The mean fibre length and the fines content followed similar trends when the experimental data was mass-weighted, surface-weighted, volume- weighted, or arithmetically weighted (data not shown).

Atomic force microscopy (AFM)

As AFM is a technique allowing investigations at a very local scale, samples containing only one type of fibres (F0: only long fibres, F100: only short fibres) were chosen for imaging. Typical images obtained by scanning these samples are shown in Fig. 7. After 15 homogenisation passes, long fibres are already highly fibrillated (Fig. 7c), whereas the sample derived from fines does not show a strong fibrillation (Fig. 7a). The difference in the degree of fibrillation is less pronounced after 35 homogenisation passes, where a 
similar fibril diameter was measured for both samples (Fig. 7 b, d). Samples derived from fines (F100) showed a much lower viscosity than those obtained from pulp (F0) (Figs. 1, 2). One can conclude that the final fibril length and the degree of fibrillation are much higher for F0 compared to F100. This supports the conclusions drawn from the viscosity measurements and the size characterizations performed with the L\&W Fiber Tester Plus.

\section{Conclusion}

Suspensions consisting of different kinds of fibres were produced and homogenised. They were then characterised by means of viscosity measurements, optical imaging, and AFM. These different approaches are complementary: rheological characterisation allowed studying the flow behaviour of homogenised suspensions, information about the fibre size distribution was then provided by the statistical calculations performed with the Fibertester. However, the resolution of this device is limited and unsuited when fibres are too small and/or highly fibrillated. This problem was partly solved by additional AFM imaging. Nevertheless, it should be stated that the AFM technique gives only very local information about a finite number of fibrils which cannot be considered as representative for the whole sample. The results of fibrillation trials using different mixtures of fines and long fibres demonstrate that fibre fines used in the present study are suitable for the production of nanofibrillated cellulose. A similar number of homogenisation passes was required for the conversion of both types of raw material into nanofibrillated cellulose, and comparable fibril diameters were obtained. A notable difference was observed with regard to the final viscosity of the fibril suspensions. Material derived from fines achieved around $60 \%$ of the viscosity value of suspensions produced from long fibres. Since all fibrils were of comparable diameters, one can conclude that the fibril length is significantly reduced in the case of fines.

Acknowledgments Open access funding provided by University of Natural Resources and Life Sciences Vienna (BOKU). Funding by the Austrian Research Promotion Agency FFG, Project No. 836650 FLIPPR (Future Lignin and Pulp Processing Resarch) is gratefully acknowledged. One of the authors (J.C.) would like to thank Christoph Hammerl for grinding, homogenising, and performing the viscosity measurements. Lukas Jagiello is thanked for the production of the fines-free samples with the pressure screen.

Open Access This article is distributed under the terms of the Creative Commons Attribution 4.0 International License (http:// creativecommons.org/licenses/by/4.0/), which permits unrestricted use, distribution, and reproduction in any medium, provided you give appropriate credit to the original author(s) and the source, provide a link to the Creative Commons license, and indicate if changes were made.

\section{References}

Agoda-Tandjawa G, Durand S, Berot S et al (2010) Rheological characterization of microfibrillated cellulose suspensions after freezing. Carbohydr Polym 80:677-686. doi:10.1016/ j.carbpol.2009.11.045

Derakhshandeh B, Kerekes RJ, Hatzikiriakos SG, Bennington CPJ (2011) Rheology of pulp fibre suspensions: a critical review. Chem Eng Sci 66:3460-3470. doi:10.1016/j.ces. 2011.04.017

Djalili-Moghaddam M, Toll S (2006) Fibre suspension rheology: effect of concentration, aspect ratio and fibre size. Rheol Acta 45:315-320. doi:10.1007/s00397-005-0021-y

Doi M, Edwards SF (1986) The theory of polymer dynamics. Oxford University Press, Oxford

Ferreira AGM, Silveira MT, Lobo LQ (2003) The viscosity of aqueous suspensions of cellulose fibres part 2. Influence of temperature and mix fibres. Silva Lusit 11:61-66

Gharehkhani S, Sadeghinezhad E, Kazi SN et al (2015) Basic effects of pulp refining on fiber properties-a review. Carbohydr Polym 115:785-803. doi:10.1016/j.carbpol. 2014.08.047

Gindl-Altmutter W, Obersriebnig M, Veigel S, Liebner F (2015) Compatibility between cellulose and hydrophobic polymer provided by microfibrillated lignocellulose. ChemSusChem 8:87-91. doi:10.1002/cssc.201402742

Henniges U, Veigel S, Lems E-M et al (2014) Microfibrillated cellulose and cellulose nanopaper from Miscanthus biogas production residue. Cellulose 21:1601-1610. doi:10.1007/ s10570-014-0232-4

Henriksson M, Henriksson G, Berglund LA, Lindström T (2007) An environmentally friendly method for enzyme-assisted preparation of microfibrillated cellulose (MFC) nanofibers. Eur Polym J 43:3434-3441. doi:10.1016/j.eurpolymj. 2007.05.038

Iotti M, Gregersen ØW, Moe S, Lenes M (2011) Rheological studies of microfibrillar cellulose water dispersions. J Polym Environ 19:137-145. doi:10.1007/s10924-0100248-2

ISO (2012) ISO 10376, Pulps-Determination of mass fraction of fines

ISO (2014) ISO 16065-2, Pulps-Determination of fibre length by automated optical analysis-Part 2: Unpolarized light method 
Iwamoto S, Lee S-H, Endo T (2013) Relationship between aspect ratio and suspension viscosity of wood cellulose nanofibers. Polym J 46:73-76. doi:10.1038/pj.2013.64

Jonoobi M, Oladi R, Davoudpour Y et al (2015) Different preparation methods and properties of nanostructured cellulose from various natural resources and residues: a review. Cellulose 22:935-969. doi:10.1007/s10570-0150551-0

Lasseuguette E, Roux D, Nishiyama Y (2008) Rheological properties of microfibrillar suspension of TEMPO-oxidized pulp. Cellulose 15:425-433. doi:10.1007/s10570007-9184-2

Leitner J, Hinterstoisser B, Wastyn M et al (2007) Sugar beet cellulose nanofibril-reinforced composites. Cellulose 14:419-425. doi:10.1007/s10570-007-9131-2

Li M-C, Wu Q, Song K et al (2015) Cellulose nanoparticles: structure-morphology-rheology relationships. ACS Sustain Chem Eng 3:821-832. doi:10.1021/acssuschemeng. $5 \mathrm{~b} 00144$

Lindström T, Naderi A, Wiberg A (2015) Large scale applications of nanocellulosic materials-a Comprehensive review. J Korea TAPPI 47:5-21. doi:10.7584/ktappi.2015. 47.6.005

Mallick PK (2008) Fiber-reinforced composites: materials, manufacturing, and design, 3rd Edition. CRC Press, Taylor \& Francis Group, Boca Raton, FL

Naderi A, Lindström T (2015) Rheological measurements on nanofibrillated cellulose systems: a science in progress. In: Mondal MIH (ed) Cellulose and cellulose derivatives: synthesis, modification and applications. Nova Science Publishers, New York, pp 187-204

Naderi A, Lindström T, Sundström J (2014) Carboxymethylated nanofibrillated cellulose: rheological studies. Cellulose 21:1561-1571. doi:10.1007/s10570-014-0192-8

Pääkko M, Ankerfors M, Kosonen H et al (2007) Enzymatic hydrolysis combined with mechanical shearing and highpressure homogenization for nanoscale cellulose fibrils and strong gels. Biomacromolecules 8:1934-1941. doi:10. 1021/bm061215p
Parkinson C, Matsumoto S, Sherman P (1970) The influence of particle-size distribution on the apparent viscosity of nonnewtonian dispersed systems. J Colloid Interface Sci 33:150-160. doi:10.1016/0021-9797(70)90082-2

Powell RL (1991) Rheology of suspensions of rodlike particles. J Stat Phys 62:1073-1094. doi:10.1007/BF01128178

Puisto A, Illa X, Mohtaschemi M, Alava M (2012) Modeling the rheology of nanocellulose suspensions. Nord Pulp Pap Res 27:277-281. doi:10.3183/NPPRJ-2012-27-02-p277-281

Rezayati Charani P, Dehghani-Firouzabadi M, Afra E, Shakeri A (2013) Rheological characterization of high concentrated MFC gel from kenaf unbleached pulp. Cellulose 20:727-740. doi:10.1007/s10570-013-9862-1

Silveira MT, Ferreira AGM, Lobo LQ (2002) The viscosity of aqueous suspensions of cellulose fibers part 1. Influence of consistency and fiber length. Silva Lusit 10:171-178

Silveira MT, Ferreira AGM, Lobo LQ (2003) The viscosity of aqueous suspensions of cellulose fibres. Part 3. Influence of pH. Silva Lusit 11:201-206

Syverud K, Chinga-Carrasco G, Toledo J, Toledo PG (2011) A comparative study of Eucalyptus and Pinus radiata pulp fibres as raw materials for production of cellulose nanofibrils. Carbohydr Polym 84:1033-1038. doi:10.1016/ j.carbpol.2010.12.066

Tanaka R, Saito T, Ishii D, Isogai A (2014) Determination of nanocellulose fibril length by shear viscosity measurement. Cellulose 21:1581-1589. doi:10.1007/s10570-014-0196-4

TAPPI (1994) T261 cm-94, Fines fraction of paper stock by wet screening

Turbak A, Snyder F, Sandberg K (1983) Microfibrillated cellulose. US Pat. 4,374,702

Veigel S, Rathke J, Weigl M, Gindl-Altmutter W (2012) Particle board and oriented strand board prepared with nanocellulose-reinforced adhesive. J Nanomater 2012:1-8. doi:10. $1155 / 2012 / 158503$

Zimmermann T, Bordeanu N, Strub E (2010) Properties of nanofibrillated cellulose from different raw materials and its reinforcement potential. Carbohydr Polym 79:10861093. doi:10.1016/j.carbpol.2009.10.045 